\title{
Die Sterilität der Bastarde im Lichte des Mendelismus.
}

\author{
Von Edmund Malinowski, Polytechnikum Warschau.
}

(Eingegangen 22. September 1918.)

Die Artbastarde sind oft in höherem oder minderem Grade steril. Sie können auch völlig steril sein. Die Sterilität der Pflanzenbastarde beruht darauf, daß sich die Pollenkörner und die Embryosäcke anormal entwickeln und zur Befruchtung unfähig sind. Die Pollenkörner besitzen sogar keine Keimungsfähigkeit. Bei völlig sterilen Bastarden entwickeln sich alle Pollenkörner und alle Embryosäcke anormal, bei den teilweise sterilen Bastarden geschieht es so nur mit einem gewissen Prozent von ihnen. Völlig sterile Bastarde entwickeln keine Samen, dagegen die teilweise sterilen Bastarde ergeben weniger Samen, als normale fertile Pflanzen.

Manchmal hängt der Sterilitätsgrad vom Geschlecht ab. Z. B. bei dem Bastarde Antirrhinum siculum $\times$ A. majus sind alle Samenanlagen steril, während der Pollen fertil ist; man kann mit ihm $A$. majus bestäuben (Baur, 1). Die Bastarde von Nicotiana Tabacum (v. atropurpurea) $\times N$. silvestris besitzen völlig sterilen Pollen und ein kleines Prozent fertiler Samenanlagen. Um Samen von dieser Pflanze zu gewinnen, muß man sie mit fremdem Pollen bestäuben, z, B. mit dem Pollen von Nicotiana silvestris (Malinowski, 4). Ähnliches Verhalten zeigt der Bastard von Weizen und Roggen (Triticum sativum $\times$ Secale cereale), welcher sterile Pollenkörner und gewisses Prozent steriler Embryosäcke besitzt (Jesenko, 3).

Teilweise sterile Bastarde können in $\mathrm{F}_{2}$ völlig fertile Nachkommenschaft geben. Bei der Kreuzung von Pisum Palestinae mit Pisum sativum bekam Sutton (3) völlig sterile Bastarde mit Ausnahme von 
zwei, die eine kleine Anzahl Samen entwickelten. Diese Samen gaben völlig fertile Nachkommenschaft. Auch die Bastarde von Erophila cochleata $\times E$. radians sind teilweise steril. In $\mathrm{F}_{2}$ dieser Bastarde beobachtete Rosen (6) eine große Variabilität in bezug auf die Fruchtbarkeit: „sie war zwar nirgends bedeutend, aber bei manchen Individuen gleichwohl gegen $F_{1}$ wieder gestiegen, bei vielen anderen auf ein Minimum herabgesunken oder völliger Sterilität gewichen". Die zweite Generation von Erophila cochleata $\times E$. radians enthielt "nicht einfach fertile und sterile Individuen, sondern alle Übergänge zwischen leidlicher Fruchtbarkeit und völligem Versagen der Fortpflanzungserscheinungen". Rosen behauptet, daß sich die Vererbungserscheinungen der partiellen Sterilität dieser Bastarde nicht in die Rahmen der Mendelschen Gesetze einschränken ließen. Im Jahre 1913 spricht er folgende Meinung aus: „Unter den $\mathrm{F}_{2}$-Pflanzen waren - im allgemeinen - diejenigen die fruchtbarsten, die am wenigsten von den Stammeltern abwichen; die extravaganten hingegẹn waren fast oder völlig steril. Diese oft wiederholte Beobachtung schien mir mit den Prinzipien der Mendelschen Spaltung schlecht vereinbar sein." (Rosen, 7.)

Bei der Kreuzung von Stizolobium deeringianum $\times S$. hassjo und $S$. deeringianum $\times S$. niveum fand Belling (2) in $F_{1}$ ein großes Prozent steriler Pollenkörner, sowie anormal ausgebildeter Embryosäcke. In $\mathrm{F}_{2}$ dieser Kreuzungen erschienen völlig fertile und teilweise sterile Pflanzen im Zahlenverhältnis, das sich zu $1: 1$ näherte. Immer jedoch waren teilweise sterile Pflanzen zahlreicher. Die von Belling für $\mathrm{F}_{2}$ gegebenen Zahlen sind folgende: 164 teilweise sterile Pflanzen auf 157 fertile im ersten Fall, und 229 teilweise sterile Pflanzen auf 169 fertile - im zweiten Fall. Belling meint, die partielle Sterilität sei bedingt durch das Zusammentreffen in den Gameten der genetischen Faktoren $\mathbf{K}$ und $\mathbf{L}$. Die Anwesenheit eines jeden von diesen Faktoren (doch nicht beider zugleich) ruft die normale Entwicklung des Pollens und der Embryosäcke hervor. Nach der Annahme von Belling besitzt die erste Bastardgeneration die genetische Zusammensetzung Kk LI und bildet vier Typen von Gameten aus: $\mathbf{K} \mathbf{L}, \mathbf{K} \mathbf{l}, \mathbf{k} \mathbf{L}, \mathbf{k} \mathbf{l}$. Von diesen vier Typen entwickeln sich zwei anormal, nämlich $\mathbf{K} \mathbf{L}$ und $\mathbf{k} \mathbf{l}$, und sind zur Befruchtung unfähig. Normale männliche, sowie weibliche Gameten sind nur von zwei Typen, nämlich $\mathbf{K} \mathbf{l}$ und $\mathbf{k} \mathbf{L}$. Theoretisch $\mathrm{mu} \mathbf{B}$ also das Verhältnis von teilweise sterilen Pflanzen zu den fertilen $1: 1$ betragen.

Die Annahme von Belling scheint mir nicht richtig zu sein. Wenn wir annehmen, daß das Zusammentreffen von $\mathbf{K}$ und $\mathbf{L}$ eine 
anormale Gametenentwicklung hervorruft, so bleibt es unerklärt, warum die Abwesenheit dieser beiden Faktoren auch eine abnorme Gametenentwicklung bedingt. Auch die gewonnenen Zahlen stimmen nicht völlig mit dem theoretischen Verhältnis $1: 1$, da im Experiment immer die teilweise sterilen Pflanzen gegen die fertilen im Übergewicht auftreten.

Auf Grund der experimentellen Angaben Bellings, sowie meiner eigenen Experimente über Weizenbastarde, habe ich eine andere Hypothese ausgearbeitet, welche uns, meiner Meinung nach, besser die Resultate der Bellingschen Experimente erklärt und welche auch die von mir bei Weizenbastarden beobachteten komplizierten Erscheinungen der partiellèn und völligen Sterilität umfaßt. Ich nehme an, die partielle oder völlige Sterilität der Bastarde sei bedingt durch das Zusammentreffen in einer Zygote von zwei oder einer größeren Anzahl von Elementen, die miteinander nicht harmonisieren.

Erörtern wir den einfachsten Fall, wenn in der Pflanze zwei solche Elemente zusammentreffen. Bezeichnen wir diese Elemente A und B. Die genetische Zusammensetzung der einen elterlichen Pflanze wird dann AAbb sein, der zweiten dagegen - aaBB. Die erste Generation wird die Konstitution AaBb haben und wird teilweise steril. Die Sterilität betrifft in gleichem Grade alle Typen von Gameten. Z. B. ein Teil der Gameten A B wird fertil und ein anderer Teil wird steril sein. Dasselbe trifft für alle anderen Gametentypen zu. Aus dem oben Gesagten geht hervor, daß fertile Gameten von folgenden vier Typen sein werden: $\mathbf{A B}, \mathbf{A} \mathbf{b}, \mathbf{a B}$, ab. Die Tabelle I stellt alle möglichen Kombinationen dieser fertilen Gameten, mit anderen Worten alle Typen der Zygoten der zweiten Generation dar. Die auf der Tabelle I

Tabelle I.

\begin{tabular}{|c|c|c|c|}
\hline $\mathrm{AB}$ & $\mathrm{Ab}$ & $\mathrm{aB}$ & $\mathrm{ab}$ \\
$\mathrm{AB}$ & $\mathrm{AB}$ & $\mathrm{AB}$ & $\mathrm{AB}$ \\
\hline $\mathrm{AB}$ & $\mathrm{Ab}$ & $\mathrm{aB}$ & $\mathrm{ab}$ \\
$\mathrm{Ab}$ & $\mathrm{Ab}+$ & $\mathrm{Ab}$ & $\mathrm{Ab}$ \\
\hline $\mathrm{AB}$ & $\mathrm{Ab}$ & $\mathrm{aB}$ & $\mathrm{ab}$ \\
$\mathrm{aB}$ & $\mathrm{aB}$ & $\mathrm{aB}$ & $\mathrm{aB}$ \\
\hline $\mathrm{AB}$ & $\mathrm{Ab}$ & $\mathrm{aB}$ & $\mathrm{ab}$ \\
$\mathrm{ab}$ & $\mathrm{ab}+$ & $\mathrm{ab}+$ & $\mathrm{ab}$ \\
\hline
\end{tabular}


mit + bezeichneten Typen sind fertil, da sie keine nicht harmonisierenden Elemente besitzen. Es treten sieben solche Typen auf die Gesamtzahl 16 auf. Die übrigen Typen, in der Zahl 9, enthalten die Elemente $\mathbf{A}$ und $\mathbf{B}$ in der Ein- oder doppelten Zahl und deshalb sind sie teilweise steril. Das Zahlenverhältnis der teilweise sterilen Pflanzen zu den völlig fertilen beträgt $9: 7$, nähert sich also mehr dem von Belling beobachteten Experimentalverhältnis, als das von diesem Autor angenommene theoretische Verhältnis $1: 1$.

Ich gehe jetzt zu einem komplizierten Beispiel über.

Wenn sich in einem Bastarde mehr als ein, eventuell zwei Paar nichtharmonisierender Elemente befinden, dann ist der Sterilitätsgrad dieses Bastardes höher oder bei einer größeren Anzahl dieser Elemente wird der Bastard völlig steril sein. Nehmen wir an, die eine zur Kreuzung benutzte Pflanze enthielte die Elemente $\mathbf{A}$ und $\mathbf{B}$, und die andere $\mathbf{C}$ und D. A harmonisiert nicht mit $\mathbf{C}$, und $\mathbf{B}$ harmonisiert nicht mit D. Wenn sich in einer Pflanze die Elemente $\mathbf{A}, \mathbf{B}, \mathbf{C}$ und $\mathbf{D}$ zusammentreffen, dann ist der Sterilitätsgrad der Pflanze höher, als wenn sie nur zwei Faktoren, z. B. A und C, enthielte. Eine Pflanze mit der genetischen Zusammensetzung A A B B CCDD zeigt einen höheren Sterilitätsgrad, als eine Pflanze mit der Zusammensetzung AaBbCcDd, oder A A b bCCdd. Wenn wir annehmen, daß die Konstitution von zwei zur Kreuzung genommenen Pflanzen AABBccdd und aabbCCDD ist, dann wird der Bastard aus dieser Kreuzung die Zusammensetzung AaBbCcDd haben. Es wird eine teilweise sterile Pflanze sein, d. h. ein gewisser Teil ihren Mikro- und Makrosporen wird anormal ausgebildet. Das soll aber nicht bedeuten, daß sich nur Mikro- und Makrosporen von bestimmter Konstitution anormal ausbilden. Im Gegenteil betrifft die anormale Entwicklung im gleichen Grade Mikro-. und Makrosporen aller Typen.

Der Bastard der ersten Generation bildet also 16 Typen von fertilen Gameten aus, nämlich: ABCD, A BCd, ABCD, A BCD, aBCD, ABcd, AbcD, abCD, aBCd, aBcD, AbCd, Abcd, aBcd, abCd, abc I), abed. In $\mathrm{F}_{2}$ finden 256 Kombinationen statt, die auf der beistehenden Tabelle II dargestellt sind.

Aus dieser Tabelle erhellt, daß auf je 256 Pflanzen der zweiten Bastardengeneration 49 völlig fertile Pflanzen erscheinen. Es sind Individuen, in welchen die nichtharmonisierenden Elemente nicht zusammentreffen. Auf der Tabelle sind diese Kombinationen mit Null (0) bezeichnet. Alle übrigen Individuen sind in höherem oder niedrigerem 
Tabelle II.

\begin{tabular}{|c|c|c|c|c|c|c|c|c|c|c|c|c|c|c|c|c|}
\hline & 亩 & $\begin{array}{l}\overrightarrow{0} \\
\end{array}$ & $\stackrel{9}{8}$ & 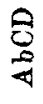 & 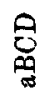 & ఫ్ర & 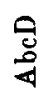 & 잉 & 己్ల్ల & 总 & $\begin{array}{l}\text { 己ु } \\
\end{array}$ & 苞 & 总 & ర్ల & ฮี & 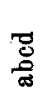 \\
\hline$A B C D$ & 4 & 3 & 3 & 3 & 3 & 2 & 2 & 2 & 2 & 3 & 3 & 2 & 2 & 2 & 2 & 2 \\
\hline $\mathrm{ABCd}$ & 3 & 2 & 2 & 3 & 2 & 1 & 2 & 2 & 1 & 2 & 2 & 1 & 1 & 1 & 2 & 1 \\
\hline$A B c D$ & 3 & 2 & 2 & 2 & 3 & 1 & 1 & 2 & 2 & 2 & 2 & 1 & 1 & 2 & 1 & 1 \\
\hline $\mathrm{AbCD}$ & 3 & 3 & 2 & 2 & 2 & 2 & 1 & 1 & 2 & 2 & 2 & 1 & 2 & 1 & 1 & 1 \\
\hline $\mathrm{aBCD}$ & 3 & 2 & 3 & 2 & 2 & 2 & 2 & 1 & 1 & 2 & 2 & 2 & 1 . & 1 & 1 & 1 \\
\hline $\mathrm{ABed}$. & 2 & 1 & 1 & 2 & 2 & 0 & 1 & 2 & 1 & 1 & 1 & 0 & 0 & 1 & 1 & 0 \\
\hline$A b c D$ & 2 & 2 & 1 & 1 & 2 & 1 & 0 & 1 & 2 & 1 & 1 & 0 & 1 & 1 & 0 & 0 \\
\hline abCD $\ldots$ & 2 & 2 & 2 & 1 & 1 & 2 & 1 & 0 & 1 & 1 & 1 & 1 & 1 & 0 & 0 & 0 \\
\hline $\mathrm{aBCd} \ldots$ & 2 & 1 & 2 & 2 & 1 & 1 & 2 & 1 & 0 & 1 & 1 & 1 & 0 & 0 & 1 & 0 \\
\hline $\mathrm{aBcD} \ldots$ & 3 & 2 & 2 & 2 & 2 & 1 & 1 & 1 & 1 & 2 & 2 & 1 & 1 & 1 & 1 & 1 \\
\hline $\mathrm{AbCd} \ldots$ & 3 & 2 & 2 & $\mathbf{2}$ & 2 & 1 & 1 & 1 & 1. & 2 & 2 & 1 & 1 & 1 & 1 & 1 \\
\hline Abed. & 2 & 1 & 1 & 1 & 2 & 0 & 0 & 1 & 1 & 1 & 1 & 0 & 0 & 1 & 0 & 0 \\
\hline $\mathrm{aBcd}$ & 2 & 1 & 1 & 2 & 1 & 0 & 1 & 1 & 0 & 1 & 1 & 0 & 0 & 0 & 1 & 0 \\
\hline $\mathrm{abCd}$ & 2 & 1 & 2 & 1 & 1 & 1 & 1 & 0 & 0 & 1 & 1 & 1 & 0 & 0 & 0 & 0 \\
\hline abcl) & 2 & 2 & l & 1. & 1 & 1 & 0 & 0 & 1 & 1 & 1 & 0 & 1 & 0 & 0 & 0 \\
\hline abed & 2 & 1 & 1 & 1 & 1 & 0 & 0 & 0 & 0 & 1 & 1 & 0 & 0 & 0 & 0 & 0 \\
\hline
\end{tabular}

Grade steril. Der Grad der Sterilität einer Pflanze, die drei oder vier Paar nichtharmonisierender Elemente enthält, ist höher, als derjenigen Pflanze, die nur zwei oder ein Paar dieser Elemente enthält. In unserem Beispiel kommen vor Pflanzen

mit 4 Paaren nichtharmonisierender Faktore in der Zahl 1,

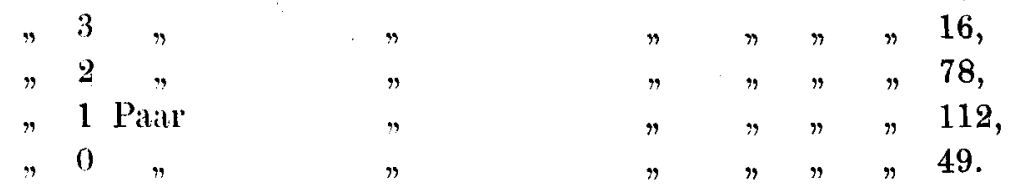

Im Bastard können auch mehr als zwei Paar nichtharmonisierender Faktoren zusammentreffen. Dann wird in $\mathrm{F}_{2}$ das Prozent völlig nnd teilweise steriler Pflanzen noch größer als im Beispiel. Solch einen komplizierten Fall beobachtete ich in der Nachkommenschaft der Bastarde von zwei Weizenarten: Triticun vulgare Vill. und Tr. dicoccum Schrk. Die erste Generation von sieben Kreuzungen, die ich in dieser Hinsicht untersucht habe, war teilweise steril. Es wurden folgende Kreuzungen ausgefïhrt:

Kreuzung Nr. 1. Q Triticum vulgare mit lockeren Ähren (Landweizen aus Wysokie Litewskie) $\times \circlearrowleft T r$. dicoccum mit mitteldichten Ähreu. 
Kreuzung Nr. 2. $\quad$ Tr. vulgare mit lockeren Ähren (Landweizen aus Wysokie Litewskie) $\times \sigma^{7} T r$. dicoccum mit lockeren Ähren.

Kreuzung Nr. 3. ㅇ $7 r$, dicoscum mit lockeren Ähren $\times \delta^{\top} T r$. vulgare mit mitteldichten Ähren (Solmanns Glatter Square head).

Kreuzung Nr. 4. + Tr. vulgare mit mitteldichten Ähren (Solmanns Glatter Square head $\times \sigma^{\top} T r$. dicoccum mit lockeren Ähren.

Kreuzung Nr. 5. + Tr. vulgare mit lockeren Ähren (Landweizen aus Wysokie Litewskie) $\times \sigma^{7} T r$. dicoccum mit mitteldichten Ähren. Kreuzang Nr. 6. $\quad$ T Tr, vulgare mit lockeren Ähren (Landweizen aus Wysokie Litewskie) $\times \sigma^{7} T r$. dicoccum mit lockeren Ähren.

Kreuzung Nr. 7. ㅇ Tr. vulgare mit lockeren Ähren (Landweizen aus Sandomir) $\times o^{T} T r$. dicoccum mit lockeren Ähren.

Den Fertilitätsgrad der Pflanzen bestimmte ich auf diese Weise, daß ich nach der Berechnung in der am besten ausgebildeten Ähre jeder Pflanze der Zahl der Ährchen und der Körner, diese letzte durch die erste dividierte. Wenn z. B. die Zahl der Ährchen in der Ähre 20 und die Zahl der Körner 15 beträgt, so ist der Fertilitätsgrad $15: 20=0,7$. Falls in der Ähre überhaupt keine Körner sich entwickeln, dann ist der Fertilitätsgrad 0. Die Tabelle III stellt Zahlen dar, welche den Fertilitätsgrad der Pflanzen der ersten Generation der eben erwähnten sieben Kreuzungen ausdrücken.

Tabelle III.

\begin{tabular}{c|r|r|r|r|r|r|r|r|r|r|r|r|r|r|r|r}
\hline $\begin{array}{c}\text { Kreuzung } \\
\text { Nr. }\end{array}$ & \multicolumn{10}{c|}{ Fertilitätsgrad der Pflanzen $F_{1}$} \\
\hline & 0,2 & 0,3 & 0,4 & 0,5 & 0,6 & 0,7 & 0,8 & 0,9 & 1,0 & 1,1 & 1,2 & 1,3 & 1,4 & 1,5 & 1,6 & 1,7 \\
\hline & & 3 & 2 & 4 & 1 & 2 & 4 & 1 & 1 & & & & & & & \\
2 & 1 & 1 & 2 & 5 & 3 & 2 & 2 & 2 & 4 & 1 & 1 & & & & & \\
3 & & & & & & & & & 2 & 3 & 4 & 3 & 3 & 2 & & \\
4 & & & & 2 & - & 1 & 2 & 1 & 3 & 1 & 6 & 2 & 1 & 1 & 1 & 2 \\
5 & & & & & 3 & - & 1 & 1 & - & 1 & & & & & & \\
6 & & & 5 & 2 & 2 & - & 3 & & & & & & & & & \\
7 & 1 & 4 & 7 & 3 & 1 & 1 & 1 & 1 & & & & & & & &
\end{tabular}

In morphologischer Hinsicht war die erste Generation von $T r$. vulgare $\times T r$. dicoccum intermediär im Verhältnis zu den elterlichen Typen. In der zweiten Bastardgeneration erschienen zahlreiche neue Formen (Malinowski, 5). Die wichtigsten morphologischen Typen der zweiten Generation waren: 1.Tr. vulgare, 2. Tr. dicoccum, 3. Tr. Spelta, 
4. Tr. durum, 5. Tr. turgidum und Formen, denen ich neue Artnamen gab, nämlich: 6. Tr. lanceolatum und 7. Tr. ellipticum. Tr. lanceolatum nähert sich mit dem Habitus der Ähre von $T r$, vulgare, besitzt aber sehr schmale und lange Hüllspelzen. Tr. ellipticum dagegen erinnert an $T r$. dicoccum mit dem Habitus der Ähre, und die Hüllspelzen dieser Art sind kurz, manchmal um die Hälfte kürzer als die Deckspelzen, und sind elliptisch. Alle diese neuen Formen der zweiten Generation erwiesen sich als erblich.

In der zweiten Generation der Kreuzungen $T r$. vulgare $\times T r$. dicoccum sind die teilweise sterilen Pflanzen viel zahlreicher als die fertilen. Es erscheint auch ein bedeutendes Prozent völlig steriler Pflanzen. Das Übergewicht von teilweise und völlig sterilen Pflanzen im Verhältnis zu den fertilen ist größer, als in dem theoretischen von mir gegebenen Beispiel. Wahrscheinlich also handelt es sich hier um eine größere Anzahl nichtharmonisierender Elemente als in jenem Beispiel. Völlig sterile Pflanzen erscheinen in dieser Generation dann, wenn sich die maximale Anzahl dieser Elemente vereinigt, oder sich ihre Anzahl zu der maximalen nähert.

Auf den Tabellen IV und $V$ sind sterile Ähren mit einer Null (0) bezeichnet, dann folgen verschiedene Grade der partiellen Sterilität von 0,1 bis $1,2-1,5$, weiter haben wir schon mit fertilen Pflanzen zu tun. Die Schwankungsgrenze der Fertilität von Tr. vulgare (Landweizen aus Wysokie Litewskie) erstreckt sich von 1,0-2,3; diejenige von $T r$. dicoccum von $1,4 \mathrm{zu} 2,5$ (Tabelle IV). Das Fertilitätsmaximum der zweiten Bastardgeneration von $T r$. vulgare $\times I r$. dicoccum war 2,8. Die Tabelle IV zeigt, wieviel Individuen auf jede Fertilitätsklasse kommen (von $0-2,8$ ) in der zweiten Generation der einzelnen Kreuzungen. Die meisten Individuen besitzen die Fertilität 0,1 , oder in manchen Kreuzungen $0,2-0,3$. Im allgemeinen sind in der zweiten Generation der erwähnten Kreuzungen teilweise sterile Pflanzen mit den Sterilen viel zahlreicher als die Fertilen.

Die Erscheinungen der gegenseitigen Abhängigkeit zwischen morphologischen Eigenschaften und der völligen oder partiellen Sterilität sind sehr undeutlich angezeigt. Alle Grundtypen (vulgare, dicoccum, Spelta, lanceolatum und ellipticum) sind ungefähr gleich in bezug auf ihre Fruchtbarkeit. Die Tabelle V stellt Zahlen für die verschiedenen Fertilitätsgrade der einzelnen $\mathrm{F}_{2}$-Typen der Bastarde von $T r$. vulgare $\times T r$. dicoccum dar. Im Gebiet des Typus Tr. lanceolatum befinden sich am zahlreichsten Pflanzen von hohem Sterilitätsgrad. 
Tabelle

\begin{tabular}{|c|c|c|c|c|c|c|c|c|c|c|c|c|}
\hline \multirow{2}{*}{$\begin{array}{c}\text { Kreuzung } \\
\mathrm{Nr} .\end{array}$} & \multicolumn{12}{|c|}{ Fertilitätsgrad } \\
\hline & 0 & 0,1 & 0,2 & 0,3 & 0,4 & 0,5 & 0,6 & 0,7 & 0,8 & 0,9 & 1,0 & 1,1 \\
\hline 1 & 8 & 8 & 8 & 11 & 9 & 11 & 11 & 9 & 16 & 5 & 9 & 12 \\
\hline 2 & 20 & 53 & 42 & 57 & 52 & 42 & 38 & 32 & 34 & 19 & 16 & 16 \\
\hline 3 & 46 & 53 & 44 & 28 & 24 & 29 & 34 & 29 & 29 & 26 & 23 & 22 \\
\hline 4 & 28 & 31 & 17 & 19 & 14 & 13 & 11 & 8 & 13 & 27 & 16 & 18 \\
\hline 5 & 1 & 5 & 1 & 4 & 3 & 2 & 2 & 1 & 5 & $\mathbf{3}$ & $\mathbf{3}$ & 5 \\
\hline 6 & 10 & 10 & 11 & 6 & 12 & 9 & 11 & 5 & 7 & 10 & 11 & 10 \\
\hline 7 & 16 & 37 & 15 & 14 & 19 & 11 & 15 & 15 & 11 & 9 & 7 & 8 \\
\hline Tr. vulgare & - & - & - & - & - & - & - & - & - & - & 1 & 1 \\
\hline Tr. dicoccum & - & - & - & - & - & - & - & - & - & - & - & - \\
\hline
\end{tabular}

Tabelle V.

\begin{tabular}{|c|c|c|c|c|c|c|c|c|c|c|c|c|c|c|c|c|c|c|c|c|c|c|c|c|}
\hline \multirow{2}{*}{ Ährentypus } & \multicolumn{24}{|c|}{ Fertilitätsgrad der Pflanzen $F_{2}$} \\
\hline & 00 & & 0,2 & & 0,4 & 0,5 & & & & 0,9 & & & 1,2 & 1,3 & & 1,5 & 1,6 & 1,7 & 1,8 & 1,9 & 2,0 & & 2,2 & \\
\hline Lanceolatum & \begin{tabular}{l|l}
3 & 1 \\
2
\end{tabular} & & 8 & 6 & 9 & & 11 & 7 & 4 & 5 & 9 & 5 & 7 & & 1 & 3 & 1 & 2 & - & & & - & - & - \\
\hline Ellipticum & 1 & 3 & 1 & 1 & 3 & $\tilde{\mathbf{0}}$ & 6 & 5 & 3 & 3 & 2 & 5 & 6 & 2 & 6 & 4 & 4 & 2 & 2 & 2 & 2 & 2 & 1 & 1 \\
\hline Vulgare & 2 & 4 & 6 & 2 & 3 & 2 & 5 & 5 & 3 & 4 & 3 & 8 & 3 & 1 & 2 & 2 & 2 & 1 & 2 & 1 & & - & - & 一 \\
\hline Spelta & - & 4 & 3 & 1 & 2 & 3 & 3 & 1 & 3 & 3 & 5 & 3 & 5 & 3 & 4 & 3 & 3 & 5 & 1 & & - & - & 1 & - \\
\hline Dicoceum & 1 & 3 & & 2 & 2 & 4 & 6 & 2 & 5 & 2 & 3 & 5 & 7 & 2 & 2 & 1 & 1 & 1 & 2 & 1 & & - & - & - \\
\hline Kleine Ähren & $15 \mid 1$ & & & & 9 & 5 & 6 & 2 & 5 & 7 & & - & 1 & - & - & & - & - & - & - & - & - & - & - \\
\hline
\end{tabular}

In der zweiten Bastardgeneration erschien eine Reihe von Pflanzen mit sehr kleinen Ähren. Diese Pflanzen erreichten nicht die normale Größe und schienen unentwickelt zu sein. Die Größe der Ähre erwies sich aber als erblich. Pflanzen mit kleinen Ähren sind entweder völlig steril oder zeichnen sich durch einen hohen Sterilitätsgrad aus. Diese kleinen Ähren gehören aber zu den verschiedenen morphologischen Typen. Ihre Spelzen sind denjenigen von Tr. vulgare, dicoccum, ellipticum oder lanceolatum am meisten ähnlich; man findet manchmal abgestumpfte Spelzen, die an Tr. Spelta erinnern. Natürlich beruht diese Ähnlichkeit auf der Spelzenform, da ihre Dimensionen viel kleiner als bei den erwähnten Arten sind.

Aus obiger Zusammenstellung erhellt, daß die Sterilität nicht mit diesem oder jenen morphologischen Typus verbunden ist, sondern eine 
IV.

\begin{tabular}{|c|c|c|c|c|c|c|c|c|c|c|c|c|c|c|}
\hline 1,2 & 1,3 & 1,4 & 1,5 & 1,6 & 1,7 & 1,8 & 1,9 & 2,0 & 2,1 & 2,2 & 2,3 & 2,4 & 2,5 & 2,6 \\
\hline 5 & 8 & 3 & 3 & 2 & 3 & - & - & - & - & 1 & - & - & - & - \\
\hline 20 & 13 & 12 & 8 & 4 & 4 & 5 & 2 & 1 & - & - & - & - & - & - \\
\hline 27 & 26 & 24 & 14 & 16 & 13 & 7 & 6 & 5 & 1 & 2 & 1 & - & - & - \\
\hline 18 & 14 & 8 & 14 & 13 & 17 & 16 & 8 & 5 & 6 & 1 & 3 & - & 2 & 2 \\
\hline 8 & 3 & 3 & 4 & 4 & 2 & 2 & 3 & - & - & - & - & - & - & - \\
\hline 12 & 5 & 4 & 3 & 2 & 4 & - & - & 2 & 1 & - & 1 & - & - & - \\
\hline 6 & 3 & 6 & 3 & - & 3 & 1 & - & - & - & - & - & - & - & - \\
\hline 2 & 8 & 10 & 19 & 16 & 32 & 22 & 5 & 2 & 1 & 1 & 1 & - & - & - \\
\hline- & - & 1 & 2 & 8 & 8 & 20 & 18 & 20 & 10 & 5 & 6 & 4 & 1 & - \\
\hline
\end{tabular}

Eigenschaft ist, welche von speziellen Faktoren (Genen) hervorgerufen ist, die ich höher "nichtharmonisierende Elemente" nannte.

Die Samen von teilweise sterilen $\mathrm{F}_{2}$-Pflanzen wurden von mir im Frühling 1917 in Mistbeeten ausgesäet, um die $F_{3}$-Generation zu bekommen. Im April wurden die jungen Pflanzen in den Boden eingepflanzt. Die Samen stammten von isolierten Ähren. Einzelne $\mathbf{F}_{3-}$ Linien erwiesen Unterschiede im Fertilitätsgrad, was die Tabelle VI (S. 234 u. 235) darstellt. Die Untersuchungsresultate der dritten Generation zeigen, daß verschiedene Grade der partiellen Sterilität erblich sind.

\section{Zitierte Literatur.}

1. Baur, Einführung in die experimentelle Vererbungslehre. Berlin, 1914.

2. Belling, The Mode of Inheritance of Semi-Sterility in the offspring of Certain Hybrid Plants. Zeitschr. f. ind. Abst.- u. Vererbgsl., 1914.

3. Jesenko, Über Getreide-Speziesbastarde (Weizen-Roggen). Zeitschr. f. ind. Abst.u. Vererbgsl., 1913.

4. Malinowski, On the appearance of new forms in the posterity of hybrids of Nicotiana atropurpurea $X$ N. silvestris. C. R. Soc. Sc. Varsovie, 1916.

5. Malinowski, Etudes sur les hybrides du Froment. Travaux Soc. Sc. Varsovie, $30,1918$.

6. Rosen, Die Entstehung der elementaren Arten von Erophila verna. Beitr. z. Biol. d. Pflanzen, 1911.

7. Rosen, Die Entstehung elementarer Arten aus Hybridisation ohne Mendelsche Spaltung. Beitr. z. Biol. d. Pflanzen, 1913.

8. Sutton, Compte rendu d'expériences de croisements faites entre le pois sauvage de Palestine et le pois de commerce. 4-me Conf. de Génétique. Paris, 1911 
Tabelle

\begin{tabular}{|c|c|c|c|c|c|c|c|c|c|c|c|c|c|c|c|c|}
\hline \multirow{2}{*}{ Nr. $F_{8}$} & \multicolumn{16}{|c|}{ Fertilitätsgrad } \\
\hline & 0 & 01 & 02 & 03 & 04 & 05 & 06 & 07 & 08 & 09 & 10 & 11 & 12 & 13 & 14 & 15 \\
\hline 25 & 4 & 5 & 7 & 3 & 5 & 7 & 4 & 4 & 7 & 2 & 2 & 2 & 3 & 1 & 2 & 2 \\
\hline 26 & - & 一 & - & - & - & 一 & - & - & - & - & - & 2 & 2 & 1 & 2 & 3 \\
\hline 27 & 4 & 3 & 2 & 2 & 2 & 1 & 1 & 2 & 2 & 2 & 1 & - & - & 1 & 1 & 2 \\
\hline 28 & 16 & 9 & 3 & 5 & 3 & 1 & 1 & 1 & 2 & 2 & - & 2 & 1 & 1 & 1 & 3 \\
\hline 34 & 5 & 8 & 4 & 9 & 4 & 8 & 6 & 8 & 11 & 4 & 10 & 8 & 5 & 3 & 7 & 7 \\
\hline 36 & 1 & 3 & 5 & 1 & - & 1 & - & 1 & 6 & - & 1 & 2 & - & 2 & 1 & 1 \\
\hline 37 & 32 & 15 & 11 & 19 & 13 & 8 & 10 & 5 & 10 & 12 & 3 & 4 & 6 & 7 & 8 & 8 \\
\hline 55 & 53 & 27 & 19 & 15 & 7 & 3 & 2 & 4 & 5 & 2 & 2 & 2 & 1 & 1 & 3 & 1 \\
\hline 66 & 3 & 1 & 3 & 1 & 1 & - & - & - & - & - & - & - & - & - & - & - \\
\hline 70 & 24 & 13 & 6 & 7 & 3 & 1 & 3 & 2 & - & 1 & 1 & $\cdot 1$ & 1 & 1 & - & 1 \\
\hline 72 & - & - & 1 & 2 & 2 & - & 3 & 1 & 1 & 4 & 1 & 1 & 2 & 2 & 2 & - \\
\hline 89 & 2 & 1 & 2 & 1 & 1 & 1 & 3 & - & 2 & 1 & 1 & 8 & 8 & 3 & 3 & $\mathbf{3}$ \\
\hline 98 & 4 & 6 & 1 & 2 & 3 & 12 & 3 & 2 & 2 & 2 & 6 & 4 & 3 & 5 & 3 & 2 \\
\hline 101 & 32 & 12 & 9 & 8 & 7 & 4 & 5 & 1 & 2 & 1 & 3 & - & 1 & - & 1 & $\mathbf{2}$ \\
\hline 103 & 9 & 4 & 5 & - & - & - & - & - & - & - & - & - & - & - & - & - \\
\hline 104 & - & - & - & - & - & 1 & 1 & 1 & $\mathbf{5}$ & 4 & 1 & 1 & - & 1 & - & - \\
\hline 111 & 10 & 3 & 2 & - & - & - & 1 & - & - & - & - & - & - & - & - & - \\
\hline 115 & 5 & 2 & 3 & 1 & $\dot{-}$ & - & - & - & - & - & - & - & - & - & -- & - \\
\hline 133 & - & - & - & - & 3 & 1 & 1 & 1 & 1 & 2 & 1 & 3 & 2 & 5 & 1 & 1 \\
\hline 140 & - & - & - & - & - & - & - & 1 & 3 & 3 & 2 & 3 & 7 & 3 & 6 & 4 \\
\hline
\end{tabular}


VI.

der Pflanzen.

\begin{tabular}{r|r|r|r|r|r|r|r|r|r|r|r|r|r|r|r|r|r|r}
\hline 16 & 17 & 18 & 19 & 20 & 21 & 22 & 23 & 24 & 25 & 26 & 27 & 28 & 29 & 30 & 31 & 32 & 33 & 34 \\
\hline 1 & - & - & - & - & - & - & - & - & - & - & - & - & - & - & - & - & - & - \\
\hline & 3 & 6 & 8 & 4 & 3 & 2 & 7 & 8 & 5 & 1 & 1 & 1 & 1 & - & - & - & - & - \\
3 & 1 & 1 & - & - & 2 & 1 & - & - & - & - & - & - & - & - & - & - & - & - \\
10 & 7 & 2 & 2 & 1 & 2 & 5 & 1 & 2 & 1 & 1 & - & - & - & - & - & - & - & - \\
1 & 1 & 7 & 8 & 4 & 5 & 8 & 6 & 11 & 6 & 4 & 7 & 2 & 2 & 3 & 1 & 1 & 2 & - \\
17 & 2 & 15 & 8 & 4 & 7 & 9 & 8 & 7 & 4 & 3 & 2 & 4 & 4 & - & - & - & - & 1 \\
1 & 1 & 1 & 2 & - & - & - & 1 & - & - & - & - & - & - & - & - & - & - & - \\
- & - & - & - & - & - & - & - & - & - & - & - & - & - & - & - & - & - & - \\
- & 1 & 1 & - & - & - & - & - & - & - & - & - & - & - & - & - & - & - & - \\
3 & 3 & 2 & - & 1 & - & - & - & - & - & - & - & - & - & - & - & - & - & - \\
8 & 6 & 10 & 7 & 3 & 2 & 1 & 1 & 1 & - & 1 & - & - & - & - & - & - & - & - \\
1 & 6 & 3 & 2 & 1 & 5 & 2 & 2 & - & - & - & - & - & 1 & 1 & - & 1 & - & - \\
- & - & - & - & - & - & - & - & - & - & - & - & - & - & - & - & - & - & - \\
- & - & - & - & - & - & - & - & - & - & - & - & - & - & - & - & - & - & - \\
- & - & - & - & - & - & - & - & - & - & - & - & - & - & - & - & - & - & - \\
- & - & - & - & - & - & - & - & - & - & - & - & - & - & - & - & - & - & - \\
\hline 2 & - & - & - & - & - & - & - & - & - & - & - & - & - & - & - & - & - & - \\
5 & 2 & 1 & - & - & - & - & - & - & - & - & - & - & - & - & - & - & - & -
\end{tabular}

